\title{
Systematic uncertainties in the AMS-02 antiproton excess
}

\section{Alessandro Cuoco ${ }^{a}$, Jan Heisig ${ }^{* b}$, Lukas Klamt $^{a}$, Michael Korsmeier ${ }^{a, c, d}$ and Michael $\mathrm{Krämer}^{a}$}

${ }^{a}$ Institute for Theoretical Particle Physics and Cosmology, RWTH Aachen University, 52056 Aachen, Germany

${ }^{b}$ Centre for Cosmology, Particle Physics and Phenomenology (CP3), Université catholique de Louvain, Chemin du Cyclotron 2, B-1348 Louvain-la-Neuve, Belgium

${ }^{c}$ Dipartimento di Fisica, Università di Torino, Via P. Giuria 1, 10125 Torino, Italy

${ }^{d}$ Istituto Nazionale di Fisica Nucleare, Sezione di Torino, Via P. Giuria 1, 10125 Torino, Italy

E-mail: cuoco@physik.rwth-aachen.de, jan.heisig@uclouvain.be,

klamtephysik.rwth-aachen.de, korsmeier@physik.rwth-aachen.de,

mkraemer@physik.rwth-aachen.de

\begin{abstract}
Global fits of cosmic-ray (CR) fluxes measured by AMS-02 provide great potential to search for exotic sources of antimatter such as dark matter (DM). Previous studies of the AMS-02 antiproton data revealed an excess compatible with DM annihilation. In this article we discuss the robustness of this finding studying two sources of systematic uncertainties affecting the result: the production cross sections for secondary antiprotons and possible correlations in the experimental data, so far not provided by AMS-02. While the former has only a mild effect on the significance of the signal (persisting at the $3 \sigma$-level), correlations in the data potentially have a strong impact. Using datadriven benchmark models for the correlations, their inclusion can greatly improve the constraining power of the data and, furthermore, enhance the significance of the DM signal up to above $5 \sigma$. The analysis reveals the importance of providing the covariance of the experimental data to fully exploit its potential.
\end{abstract}

European Physical Society Conference on High Energy Physics - EPS-HEP2019 -

10-17 July, 2019

Ghent, Belgium

${ }^{*}$ Speaker. 


\section{Introduction}

Antimatter in cosmic rays (CRs), in particular antiprotons, provide a powerful probe to search for exotic CR sources, such as self-annihilating dark matter (DM). The high accuracy of the antiproton flux measurement by the AMS-02 experiment [1] potentially allows us to detect a contribution as small as about $10 \%$ of the total antiproton flux. However, to exploit this level of accuracy theoretical and experimental systematic uncertainties have to be under control at the same level.

A state-of-the-art description of CR propagation in the Galaxy is provided by the two-zone diffusion model with convection and reaccelaration [2]. A common strategy for constraining its parameters utilizes the boron-to-carbon flux ratio $(\mathrm{B} / \mathrm{C})$ measurement assuming that heavier nuclei propagate in the same way as (anti-)protons. In the analyses discussed in the following we, however, take into account light nuclei only (namely $\bar{p}, p, \mathrm{He}$ ) constraining the $\mathrm{CR}$ propagation scenario and DM at the same time. To this end we perform a joint fit of propagation and DM parameters and derive likelihoods for the DM signal strength by profiling over the propagation parameters treated as nuisance parameters. This approach enabled us to derive strong limits on heavy DM [3]. At the same time, it revealed a preference for a DM signal, corresponding to a DM mass around 40$130 \mathrm{GeV}$ and a thermal annihilation cross section, $\langle\sigma v\rangle \sim 3 \times 10^{-26} \mathrm{~cm}^{3} / \mathrm{s}$ [4, 5]. A compatible signal has also been seen using B/C to constrain propagation [6] (see also [7]). However, given the small experimental errors, several sources of systematic uncertainties, which before could have been neglected, now become potentially relevant and, hence, require further investigations.

As pointed out in [7] a potentially important source of uncertainty is given by the antiproton cross sections, needed to model the production of secondary antiprotons through scattering of CR protons and helium with the interstellar medium (ISM) in the Galactic disk. Another important source of uncertainties arises due to possible correlations in the AMS-02 data which are expected to be present, but could not been taken into account in the above analyses, as they are not provided by the collaboration. In this article we present a systematical study of these two sources of uncertainties and investigate their impact on the significance of the above-mentioned DM signal and, hence, scrutinizing the robustness of this finding [8]. Meanwhile, similar analyses have also been carried out by other groups $[9,10]$.

The article is structured as follows. In Sec. 2 we describe our numerical setup and review the DM excess found. In Sec. 3 we study the impact of uncertainties in the antiproton cross sections, incorporating uncertainties via a covariance matrix method as well as a joint fit of propagation and cross-section parameters. In Sec. 4 we discuss the potential impact of correlated errors in the AMS-02 data using several data-driven benchmark scenarios for realistic correlations in the data. We conclude in Sec. 5.

\section{Cosmic-ray propagation and default setup}

We describe the propagation of CRs through the Galaxy in the two-zone diffusion model [2]. The corresponding diffusion equations for the phase-space densities of CRs are solved with the GALPROP code [11, 12]. Diffusion is modeled with a power law in rigidity. We include convective winds assumed to be constant and orthogonal to the Galactic plane as well as diffusive reacceler- 

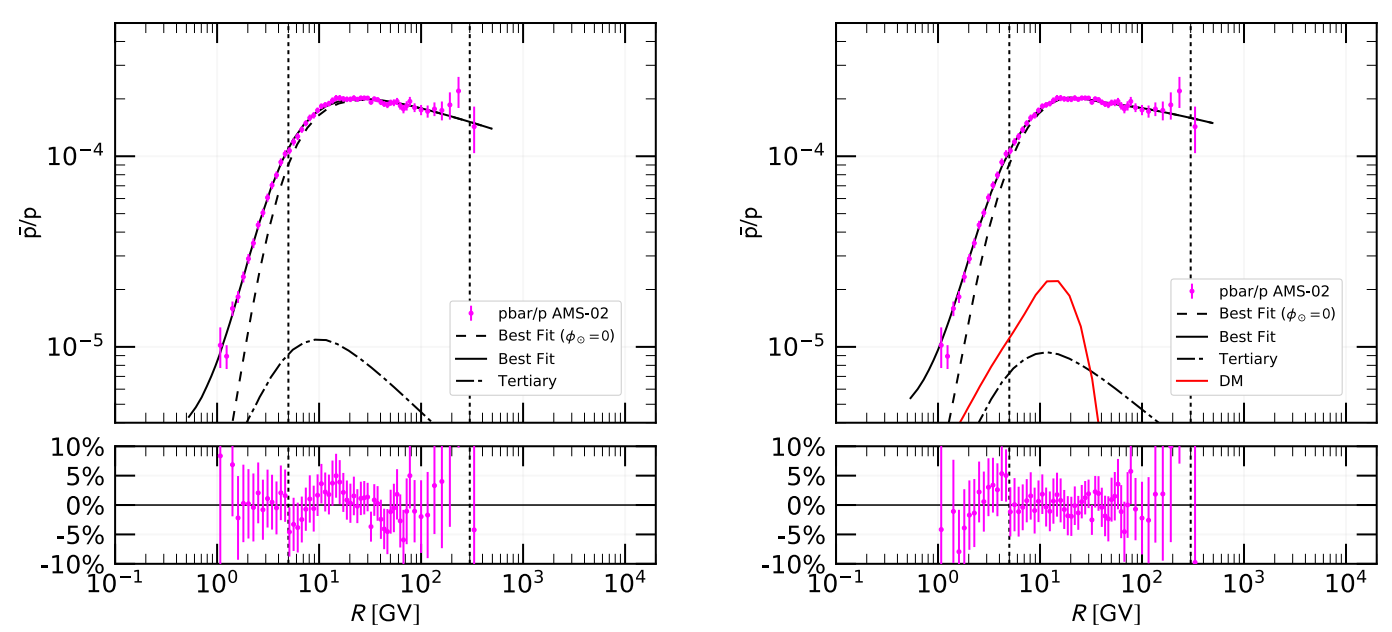

Figure 1: Comparison of antiproton-over-proton ratio for the respective best-fit points. The left (right) plot shows the case without (with) DM. In addition, we show the tertiary component, the DM component and the flux before solar modulation. We fit data in the range $R=(5-300) \mathrm{GV}$ (between the dotted lines).

ation. We model the source terms for the primary CRs by a smoothly broken power law with a common break position but individual slopes for proton and helium.

For the source term for secondary antiprotons from scatterings of CR proton and helium in the ISM we use the antiproton production cross sections from Winkler [13] with updated parameters from Korsmeier et al. [14] (see Sec. 3 for more details). Furthermore, we take into account DM annihilation as a primary source of antiprotons parametrized by the DM mass, $m_{\mathrm{DM}}$, and velocityaveraged annihilation cross section, $\langle\sigma v\rangle$. As a benchmark we consider annihilation into $b \bar{b}$. We expect the qualitative results to apply for other annihilation channels providing similar antiproton spectra (see e.g. [5]). We assume that DM is distributed according to a Navarro-Frenk-White density profile [15]. We expect little dependence on the chosen density profile as indicated by the results of [3].

In our analysis we use the AMS-02 measurements of the antiproton-to-proton ratio [1] and the proton and helium fluxes $[16,17]$. We model solar modulation using the force-field approximation [18]. The corresponding solar modulation potential is constrained by additional proton and helium data from Voyager [19] measuring directly the local interstellar flux. To account for possible charge dependent effects we allow for slightly different solar modulation potentials for antiprotons and proton/helium, however, penalizing their difference by a Gaussian prior with a variance $\sigma_{\varphi}=100 \mathrm{MV}$. As we observe a break-down of the force-field approximation towards small rigidities [8] we only fit data above $5 \mathrm{GV}$.

We perform global fits of the propagation (and DM parameters) with and without a primary source of antiprotons from DM using MULTINEST [20] for the parameter space sampling. Figure 1 shows the antiproton-over-proton ratios as function of rigidity together with the AMS-02 data for the corresponding best-fit points. Allowing for a DM contribution to CR antiprotons improves the fit by $\Delta \chi^{2}=12.7$ which, formally, corresponds to a significance of $3.1 \sigma .{ }^{1}$ The (propagated) best-fit

\footnotetext{
${ }^{1}$ Note that this significance is lower than the one originally found in [4] which was around $4.5 \sigma$. The difference is mainly due to the different antiproton cross sections chosen. In [4] the parametrizations from [21] was used.
} 

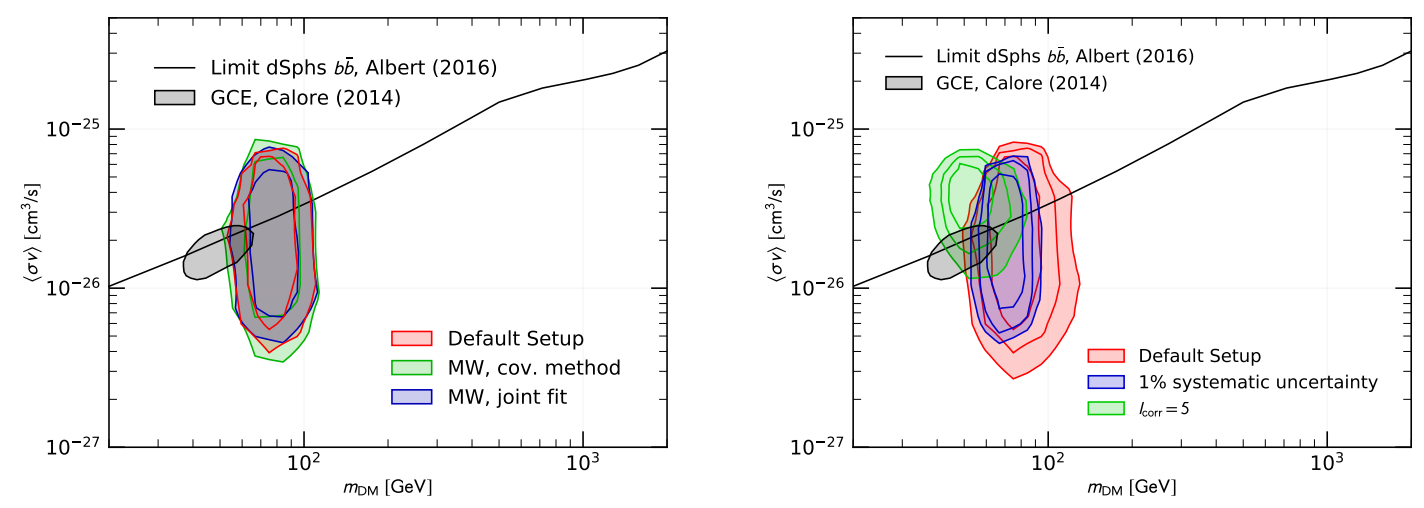

Figure 2: Best-fit regions in the $m_{\mathrm{DM}}-\langle\sigma v\rangle$ for annihilation into $b \bar{b}$. The red contours in both panels show the $1-2 \sigma(1-3 \sigma)$ regions around the best-fit point for our default setup, as described in Sec. 2. Furthermore, both panels show the 95\% CL upper limit on the annihilation cross section derived from the observation of dwarf spheroidal galaxies [22] and the $2 \sigma$ best-fit region of the GCE from [23]. Left panel: Best-fit region taking into account antiproton cross-section uncertainties via the covariance matrix approach (green contours) and the joint fit (blue contours). Right panel: Best-fit region for two benchmark scenarios for the correlations in the AMS-02 data: $1 \%$ fixed systematic uncertainties (blue contours) and $\ell_{\text {corr }}=5$ (red contours).

DM spectrum peaks at around $(10-20) \mathrm{GV}, c f$. red curve in the right panel of Fig. 1.

The corresponding $(1-3) \sigma$ contours in the $m_{\mathrm{DM}^{-}}\langle\sigma v\rangle$ plane are shown in Fig. 2 (labeled as 'default setup'). As already pointed out in [4] the best-fit region is compatible with dark matter interpretation of the gamma-ray Galactic center excess (GCE) and limits from gamma-ray observation of dwarf spheroidal galaxies.

\section{Uncertainties in the antiproton production cross sections}

The antiproton production cross section can have an important impact on the significance of the DM signal found. In this section we investigate the subject systematically by taking into account the uncertainties in the antiproton production cross sections in the fit. For a given CR projectile nucleus $i$ with flux $\phi_{i}$ and nuclei component of the ISM $j$ with density $n_{\mathrm{ISM}, j}$ the corresponding energy-differential cross section for antiproton production, $\mathrm{d} \sigma_{i, j} / \mathrm{d} T_{\bar{p}}$, enters the secondary antiproton source term in the following way:

$$
q_{i j}\left(\boldsymbol{x}, T_{\bar{p}}\right)=\int_{T_{\mathrm{th}}}^{\infty} \mathrm{d} T_{i} 4 \pi n_{\mathrm{ISM}, j}(\boldsymbol{x}) \phi_{i}\left(T_{i}\right) \frac{\mathrm{d} \sigma_{i j}}{\mathrm{~d} T_{\bar{p}}}\left(T_{i}, T_{\bar{p}}\right),
$$

where the parameters $T_{i}$ and $T_{\bar{p}}$ denote the kinetic energy of the CR projectile and the produced antiproton, respectively. We use the cross section parametrization from [13] and parameters that were updated [14] using the most recent data from NA61 and LHCb. We follow two approaches.

First, we use the covariance matrix of the cross-section parameters fitted in [14] and propagate the error to the flux of the CR antiprotons by Monte Carlo sampling. This way we obtain a covariance matrix for the antiproton flux measured by AMS-02 that is added to the one from the 
(uncorrelated) uncertainties of the AMS-02 measurement:

$$
\mathscr{V}_{i j}^{\left(\phi_{\overline{\bar{p}} / p}^{\mathrm{AMS}-02}\right)}=\mathscr{V}_{\mathrm{XS}, i j}^{\left(\phi_{\overline{\bar{p}} / p}^{\mathrm{AMS}-02}\right)}+\delta_{i j}\left[\sigma_{\bar{p} / p, i}^{(\mathrm{AMS}-02)}\right]^{2},
$$

where $i, j$ refer to the rigidity bins. A similar approach has been followed in [7].

As a second approach we perform a simultaneous fit of CR and cross-section parameters. To keep the total number of parameters on a computationally feasible level, we take into account the three most important cross-section parameters only, namely the parameters $C_{1}$ and $C_{5}, C_{6}$ in [14] which determine the over-all normalization and the shape at low energies, respectively. The remaining parameters are set to their best-fit value.

Within these two setups we perform global fits with and without DM each. ${ }^{2}$ In both setups we found a preference for DM with a similar significance, namely $\Delta \chi^{2}=10.9$ using the covariance matrix and $\Delta \chi^{2}=10.7$ performing the joint fit, which corresponds to 2.9 and $2.8 \sigma$, respectively. This implies that the inclusion of systematic uncertainties in the antiproton cross sections do not have a strong effect on our results. Furthermore, the best-fit regions in the DM mass and the velocity-averaged annihilation cross section shown in the left panel of Fig. 2 hardly change when considering cross-section uncertainties. Finally, we find that the $\chi^{2}$ profiles of the cross-section parameters in the joint fit are approximately Gaussian-like and that there are no significant correlations between the propagation and cross-section parameters. A posteriori, this justifies the use of the covariance matrix method which builds upon this assumption.

\section{Correlation in the AMS-02 data}

In the rigidity region of the excess (around $10-20 \mathrm{GV}$ ) systematic uncertainties in the AMS02 data dominate over the statistical ones. In general, these systematics are expected to be subject to sizeable correlations in rigidity. However, this information is not provided by the AMS-02 collaboration and, hence, in the above analyses we simply added statistic and systematic uncertainties in quadrature. Here we illustrate the effect of possible correlations that were neglected before considering several benchmark scenarios.

First, we assume only $1 \%$ of the systematics to be uncorrelated while the rest is assumed to be fully correlated in rigidity amounting to a freedom in the total normalization. Secondly, we aim to constrain correlations from the data itself using standard statistical inference (see [8] for details). To this end we allow the covariance matrix of the CR datasets to be the sum of three parts:

$$
\mathscr{V}=\mathscr{V}_{\text {stat }}+\mathscr{V}_{\text {short }}+\mathscr{V}_{\text {long }}
$$

Here $\mathscr{V}_{\text {stat }}$ contains all uncorrelated uncertainties (here statistic error only), $\mathscr{V}$ long describes the longrange correlations (fully correlated) while $\mathscr{V}_{\text {short }}$ describes the correlation of up to a few neighboring rigidity bins. We model the latter with the ansatz:

$$
\mathscr{V}_{\text {short }, i j}=\exp \left(-\frac{|i-j|^{\alpha}}{\ell_{\text {corr }}^{\alpha}}\right) f^{2} \sigma_{\text {sys }, i} \sigma_{\mathrm{sys}, j}
$$

\footnotetext{
${ }^{2}$ As an additional setup, in [8] we also consider the parametrization from [24] (with updated parameters from [14]) yielding similar conclusions.
} 
where $i, j$ refer to the rigidity bins and $\alpha, \ell_{\text {corr }}$ and $f$ are free parameters of our model. As $\ell_{\text {corr }}$ cannot be constrained from data, we choose several benchmark values. In the following we consider the case $\ell_{\text {corr }}=5$, while $\alpha$ and $f$ is chosen to be consistent with data. The remaining systematic uncertainty is assumed to be fully correlated, i.e. to belong to $\mathscr{N}$ long.

For these benchmark scenarios correlations have a large effect on our fit. First, we find that the fraction of totally correlated errors is sizeable. They amount to a freedom in the over-all normalization of the spectrum which is, however, already considered in the fit and, thus, does not introduce additional freedom, effectively reducing the uncertainties. The constraining power of the data is thus increased. This is further reflected in the smaller best-fit regions, $c f$. right panel of Fig. 2. The regions are slightly shifted towards smaller DM masses, and they are still compatible with the best-fit region for the GCE. Secondly, the significance of the signal increases. For the case of $1 \%$ uncorrelated uncertainty (with the rest fully correlated) we find a $\Delta \chi^{2}=30.0$ corresponding to 5.1 $\sigma$. Using the ansatz Eqs. (4.1) and (4.2) with $\ell_{\text {corr }}=5$ we obtain $\Delta \chi^{2}=17.6$, i.e. $3.8 \sigma$. Note that these results only illustrate the importance of a proper treatment of correlated uncertainties. A final conclusion can only be drawn knowing the true correlations in the AMS-02 data.

\section{Conclusion}

We discussed the importance of two sources of systematic uncertainties in the interpretation of global CR fits to the precise AMS-02 data, in particular the hint for a possible DM signal. First, we studied the uncertainties of the antiproton production cross sections affecting the source terms of secondary antiproton - the background for DM. We followed two approaches, describing the uncertainties by a covariance matrix as well as performing a joint fit of CR and cross-section parameters. Both descriptions led to comparable results. The significance of the DM signal is not strongly affected by antiproton cross-section uncertainties and persists at the $3 \sigma$-level.

Secondly, we studied the impact of potential correlations in the AMS-02 data which are expected to be present but not provided by the collaboration yet. Assigning some of the systematic uncertainties to be fully correlated effectively reduces their impact in the fit and hence increases the constraining power. Besides a shrinking of the best-fit regions, the significance of the DM signal increases, up to around $5 \sigma$. This illustrates the importance of the knowledge of the true covariance for assessing the significance of the excess and exploiting the full potential of the AMS-02 data.

\section{References}

[1] AMS collaboration, M. Aguilar et al., Antiproton Flux, Antiproton-to-Proton Flux Ratio, and Properties of Elementary Particle Fluxes in Primary Cosmic Rays Measured with the Alpha Magnetic Spectrometer on the International Space Station, Phys. Rev. Lett. 117 (2016) 091103.

[2] A. W. Strong, I. V. Moskalenko and V. S. Ptuskin, Cosmic-ray propagation and interactions in the Galaxy, Ann. Rev. Nucl. Part. Sci. 57 (2007) 285-327, [a stro-ph/0 01517 ].

[3] A. Cuoco, J. Heisig, M. Korsmeier and M. Krämer, Constraining heavy dark matter with cosmic-ray antiprotons, JCAP 1804 (2018) 004, [1711.05274].

[4] A. Cuoco, M. Krämer and M. Korsmeier, Novel Dark Matter Constraints from Antiprotons in Light of AMS-02, Phys. Rev. Lett. 118 (2017) 191102, [1610.03071]. 
[5] A. Cuoco, J. Heisig, M. Korsmeier and M. Krämer, Probing dark matter annihilation in the Galaxy with antiprotons and gamma rays, JCAP 1710 (2017) 053, [1 704.08258 ].

[6] M.-Y. Cui, Q. Yuan, Y.-L. S. Tsai and Y.-Z. Fan, Possible dark matter annihilation signal in the AMS-02 antiproton data, Phys. Rev. Lett. 118 (2017) 191101, [1610 . 03840 ].

[7] A. Reinert and M. W. Winkler, A Precision Search for WIMPs with Charged Cosmic Rays, JCAP 1801 (2018) 055, [1712.00002].

[8] A. Cuoco, J. Heisig, L. Klamt, M. Korsmeier and M. Krämer, Scrutinizing the evidence for dark matter in cosmic-ray antiprotons, Phys. Rev. D99 (2019) 103014, [1903.01472].

[9] I. Cholis, T. Linden and D. Hooper, A Robust Excess in the Cosmic-Ray Antiproton Spectrum: Implications for Annihilating Dark Matter, 1903.02549.

[10] S.-J. Lin, X.-J. Bi and P.-F. Yin, Investigating the dark matter signal in the cosmic ray antiproton flux with the machine learning method, 1903.09545.

[11] A. W. Strong, I. V. Moskalenko and O. Reimer, Diffuse continuum gamma-rays from the galaxy, Astrophys. J. 537 (2000) 763-784, [astro-ph/9811296].

[12] A. W. Strong, Recent extensions to GALPROP, 1507.05020.

[13] M. W. Winkler, Cosmic Ray Antiprotons at High Energies, JCAP 1702 (2017) 048, [1 701.04866 ].

[14] M. Korsmeier, F. Donato and M. Di Mauro, Production cross sections of cosmic antiprotons in the light of new data from the NA61 and LHCb experiments, Phys. Rev. D97 (2018) 103019, [1802.03030].

[15] J. F. Navarro, C. S. Frenk and S. D. M. White, The Structure of cold dark matter halos, Astrophys. J. 462 (1996) 563-575, [astro-ph/9508025].

[16] AMS collaboration, M. Aguilar et al., Precision Measurement of the Proton Flux in Primary Cosmic Rays from Rigidity 1 GV to 1.8 TV with the Alpha Magnetic Spectrometer on the International Space Station, Phys. Rev. Lett. 114 (2015) 171103.

[17] AMS collaboration, M. Aguilar et al., Precision Measurement of the Helium Flux in Primary Cosmic Rays of Rigidities 1.9 GV to 3 TV with the Alpha Magnetic Spectrometer on the International Space Station, Phys. Rev. Lett. 115 (2015) 211101.

[18] L. A. Fisk, Solar Modulation and a Galactic Origin for the Anomalous Component Observed in Low-Energy Cosmic Rays, Astrophys. J. 206 (1976) 333-341.

[19] E. C. Stone et al., Voyager 1 Observes Low-Energy Galactic Cosmic Rays in a Region Depleted of Heliospheric Ions, Science 341 (2013) .

[20] F. Feroz, M. P. Hobson and M. Bridges, MultiNest: an efficient and robust Bayesian inference tool for cosmology and particle physics, Mon. Not. Roy. Astron. Soc. 398 (2009) 1601-1614, [0 809.3437 ].

[21] L. C. Tan and L. K. Ng, Calculation of the equilibrium anti-proton spectrum, J. Phys. G9 (1983) $227-242$.

[22] FERMI-LAT, DES collaboration, A. Albert et al., Searching for Dark Matter Annihilation in Recently Discovered Milky Way Satellites with Fermi-LAT, Astrophys. J. 834 (2017) 110, [1611. 03184 ].

[23] F. Calore, I. Cholis, C. McCabe and C. Weniger, A Tale of Tails: Dark Matter Interpretations of the Fermi GeV Excess in Light of Background Model Systematics, Phys. Rev. D91 (2015) 063003, [1411.4647].

[24] M. di Mauro, F. Donato, A. Goudelis and P. D. Serpico, New evaluation of the antiproton production cross section for cosmic ray studies, Phys. Rev. D90 (2014) 085017, [1408 . 0288]. 